\title{
Very large aortic pseudoaneurysm repair using a septal occluder
}

\author{
Paulo R.A. Caramori*1 ${ }^{* 1}$ Ricardo Lasevitch ${ }^{1}$, Maurício L. Suksteris², Ricardo M. Piantá ${ }^{2}$, João B. Petracco ${ }^{2}$, Fabiano \\ Ramos $^{3}$, Fábio M. Velho ${ }^{4}$, Felipe S. Torres ${ }^{5}$ \\ ${ }^{1}$ Department of Cardiology, Hospital São Lucas of Pontifical Catholic University of Rio Grande do Sul, Porto Alegre, Brazil \\ ${ }^{2}$ Department of Cardiovascular Surgery, Hospital São Lucas of Pontifical Catholic University of Rio Grande do Sul, Porto Alegre, \\ Brazil \\ ${ }^{3}$ Department of Infectology, Hospital São Lucas of Pontifical Catholic University of Rio Grande do Sul, Porto Alegre, Brazil \\ ${ }^{4}$ Department of Echocardiography, Hospital São Lucas of Pontifical Catholic University of Rio Grande do Sul, Porto Alegre, \\ Brazil \\ ${ }^{5}$ Department of Medical Imaging, University of Toronto, Toronto General Hospital, Ontario, Canada
}

Received: March 24, 2021

DOI: $10.5430 /$ dcc.v8n2p14
Accepted: May 16, 2021

URL: https://doi.org/10.5430/dcc.v8n2p14

\begin{abstract}
A 63-year-old man, with previous coronary artery bypass surgery presented with infectious aortitis and a large ascending aorta pseudoaneurysm, causing compression of the left internal mammary graft. Percutaneous intervention with stenting of the graft followed by closure of the pseudoaneurysm using an atrial septal defect occluder was successfully carried out.
\end{abstract}

Key Words: Ascending aorta, Pseudoaneurysm, Percutaneous repair, Septal occluder

\section{INTRODUCTION}

Ascending aortic pseudoaneurysms (AAPs) are a rare complication in patients submitted at cardiac surgery. The incidence of AAPs has been reported to be about $0.5 \%$ and in up to $60 \%$ of aortic surgeries, they occur at the aortic cannulation, aortotomy site or at the aortic anastomosis ${ }^{[1,2]}$ and are associated with a high-risk of rupture, thrombosis, embolization and death, if left untreated. ${ }^{[3]}$

Surgical repair of AAP is its ligation or replacement of the ascending aorta with a graft. However, in patients considered high-risk for conventional surgery, catheter-based therapies coming appear alternatives less invasive and successfully for the AAPs. ${ }^{[3]}$
We would like to present a case of patient submitted a coronary artery bypass surgery complicated for mediastinitis and AAP. He was considered high-risk to surgical correction and therefore a therapeutic approach with Amplatzer plug was utilized successfully for his treatment.

\section{Case presentation}

A 63-year-old male patient presented to the emergency department with fever and asthenia. He had a history of hypertension, diabetes, and coronary artery bypass graft surgery 2 years earlier, complicated by sternal wound infection requiring prolonged antibiotic therapy with favorable outcome. However, 6 months later the patient underwent resection of the distal sternum and steel wires due to infection recurrence

*Correspondence: Paulo R.A. Caramori; Email: caramori@ cardiarte.com.br; Address: Av. Ipiranga, 6690, Room 320, 90610-000, Porto Alegre, Brazil. 
followed by antibiotic therapy.

In the current presentation, the patient had fever and chills for approximately a month and lost several pounds. He also reported progressive effort chest tightness and shortness of breath. He sought treatment in an outpatient clinic and was given oral antibiotics with some improvement of symptoms. He was referred to our center after fever relapsed.

At physical examination a pulsatile mass was readily observable at left esternal border. Initial laboratory results revealed leukocytosis (33.800 leukocytes $/ \mathrm{mm}^{3}$ ) and elevated C-reactive protein $(186 \mathrm{mg} / \mathrm{dl})$. A transesophageal echocardiography indicated a large AAP. There were no cardiac vegetations or other findings of endocarditis. Computed angiotomography (CT) revealed a pseudoaneurysm at the anterior aortic wall, at the transition of the ascending aorta to aortic arch, which measured $6.2 \mathrm{~cm} \times 4.9 \mathrm{~cm} \times 6.4 \mathrm{~cm}$, with a very large $2.6 \mathrm{~cm}$ neck as shown in Figures 1A-D. Blood stream cultures were positive for Pseudomonas aeruginosa. Antibiotics were started.

Coronary angiography showed a very severe and long stenosis of the body of the left internal mammary artery (LIMA) graft to the left anterior descendent coronary artery, that was felt be secondary to external compression caused by the growing pseudoaneurysm. After heart team discussion, patient was deemed not eligible for open chest reoperation due to high surgical risk secondary to previous complicated mediastinitis and ongoing infectious aortitis.
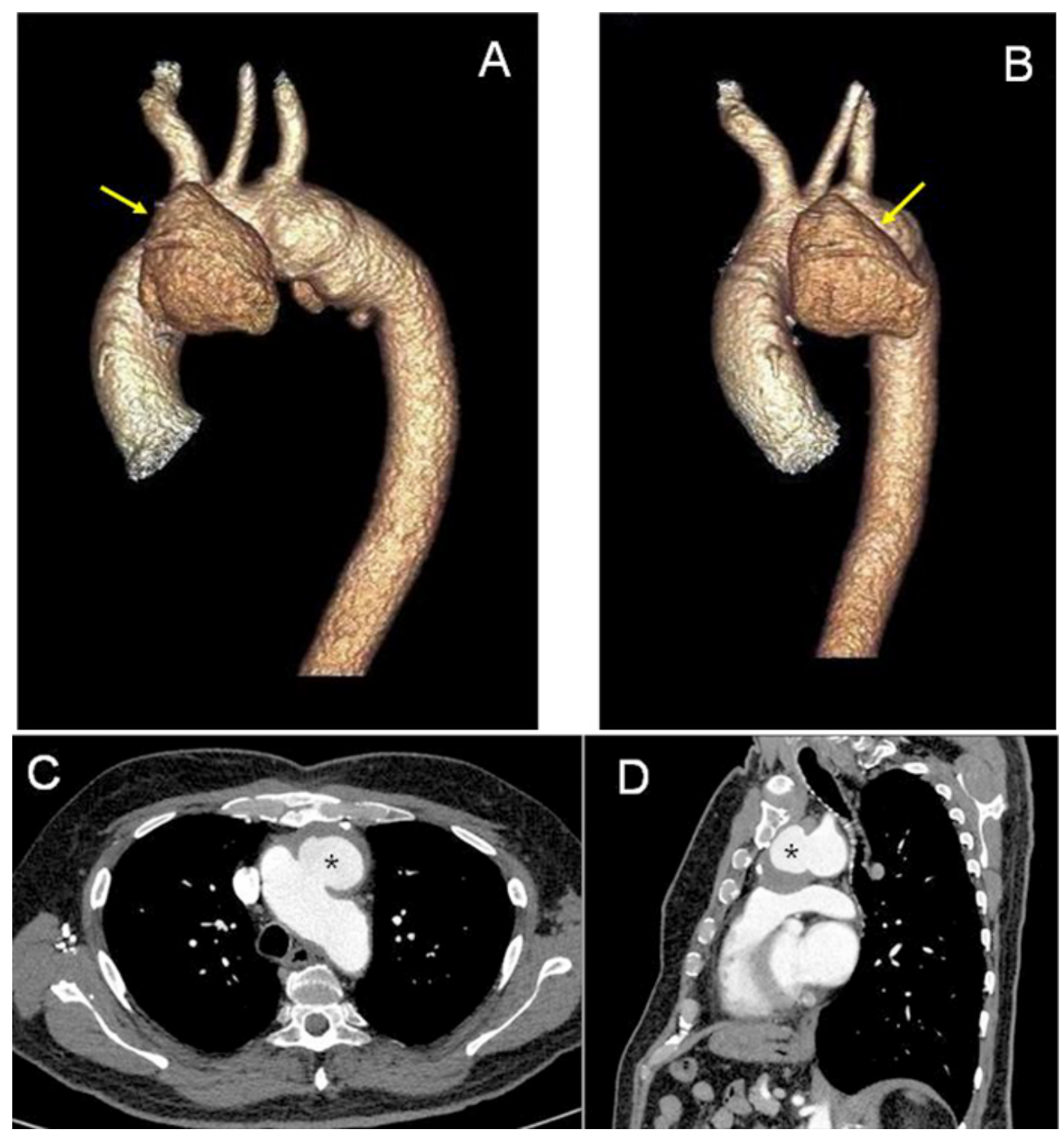

Figure 1. Large Aortic Pseudoaneurysm

A-B: 3D CT re-format of pre-procedure contrast enhanced computed tomography images demonstrating a large pseudoaneurysm (yellow arrows); $C$ : Transaxial; and D: Sagittal oblique contrast enhanced computed tomography images demonstrating a large pseudoaneurysm of the thoracic aorta (asterisks). 


\section{Management}

Initially, percutaneous intervention was successfully performed with drug eluting stents in the LIMA graft. Intravenous antibiotics were maintained for 10 weeks, with clinical and laboratory indications of infection control. A repeat CT demonstrated pseudoaneurism was stable in size. It was elected to postponed the endovascular pseudoaneurysm treatment was for a 3-month period to ensure no relapse of the Pseudomonas infection.
Endovascular pseudoaneurysm closure was performed with a CeraFlex ${ }^{\mathrm{TM}}$ ASD Occluder $28 \mathrm{~mm}$ device (Lifetech Scientific Co, Shenzhen, China), though a 12-F SteerEase ${ }^{\mathrm{TM}}$ sheath via femoral access under fluoroscopy and transesophageal echocardiography (see Figures 2A and 2B). After 18 months follow-up, the patient remains asymptomatic and in good clinical conditions. Postoperative CT confirmed exclusion and reduction in the size of the large pseudoaneurysm, despite of an initial minor endoleak, which completely resolved after 15 months (see Figures $2 \mathrm{C}$ and 2D).

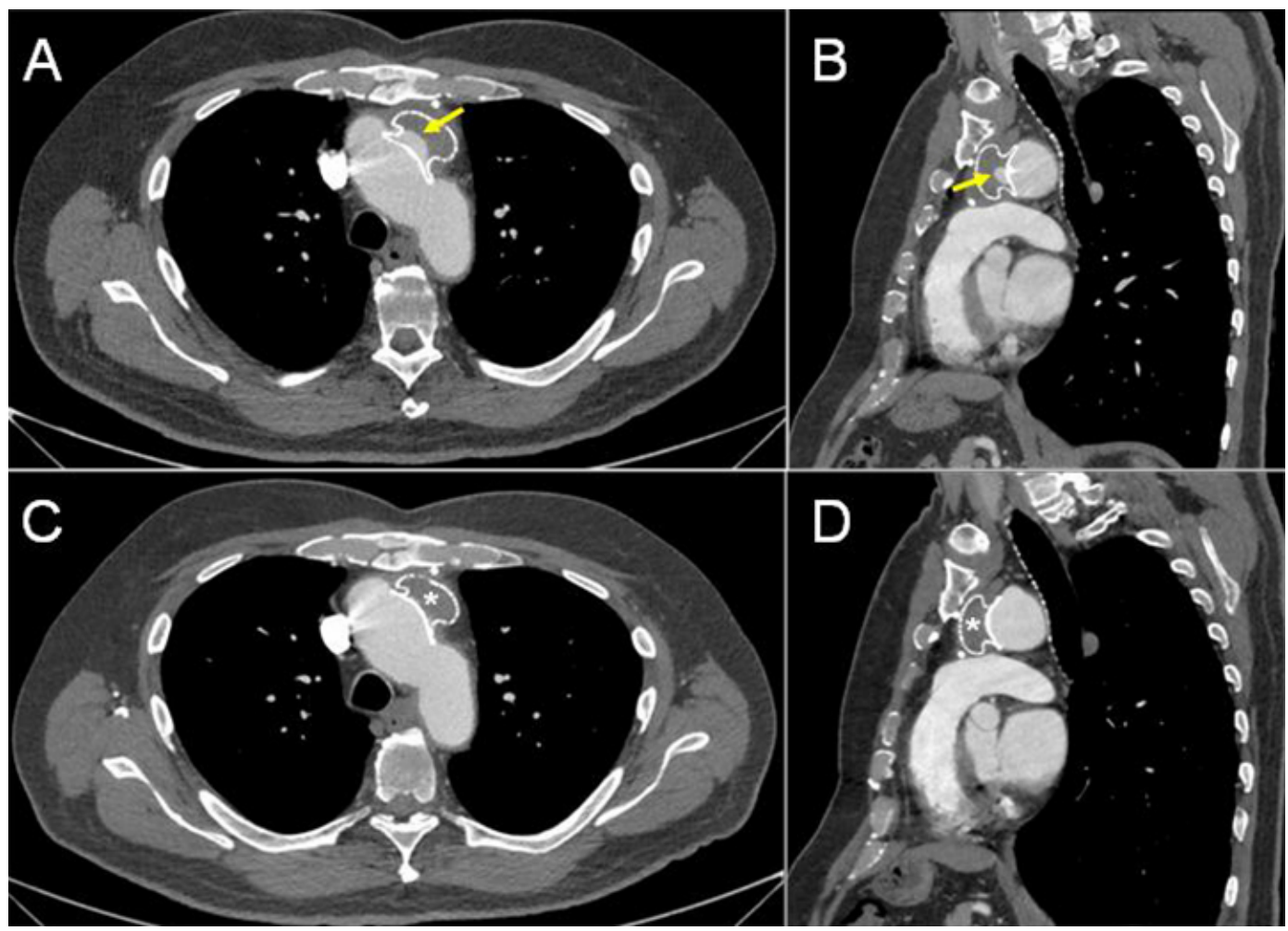

Figure 2. Evolution of pseudoaneurysm repair with septal occluder device

Nine-month $(A, B)$ and 15 months $(C, D)$ follow-up after endovascular pseudoaneurysm repair. A, C: Transaxial and B, D: Sagittal oblique contrast enhanced computed tomography images demonstrating the exclusion and reduction in the size of the large pseudoaneurysm of the thoracic aorta. A small endoleak was initially demonstrated (yellow arrows in A and B), which completely resolved after 15 months (asterisks in $C$ and $D$ ).

\section{DiscuSSION}

Aortic pseudoaneurysms are rare complications following cardiac surgery and usually occur in areas with graft anastomosis, aortomy, clamping site, neddle site or cannulation site. Less common causes include any traumatic, inflammatory or infectious event. Its incidence is about $0.5 \% .^{[1-3]}$

AAPs may progress to fatal rupture, bleeding fistula, compression or erosion of the surrounding structures. Open surgical correction is a challenge, because of the risk of AAP rupture at the reesternotomy and is associated with mortality rates of $6.7 \%-41 \%$ in different series. There aren't studies compared surgical treatment vs. endovascular repair. ${ }^{[1,2]}$

An alternative for high-risk patients is a less invasive treatment by endovascular approach. The stent grafts have several technical and anatomical challenges for treatment of ascending aorta, in addition of the current lack of specifically designed devices. ${ }^{[1,4]}$ Endovascular treatment in this setting has been expanding with others options, such as percutaneous coil embolization, direct thrombin injection and off-label use of septal occluder devices and vascular plugs. ${ }^{[1,4]}$

The first use of a septal occluder for the treatment of aortic pseudoaneurysm was reported by Bashir et al. in 2005. ${ }^{[5]}$ 
Lyen et al. had revised 34 cases of closure of aortic pseudoaneurysm with occluder devices. Device migration requiring surgery occurred in $12 \%$ of cases, while $18 \%$ presented endoleak and $6 \%$ needed surgery for endoleak correction. The use of occluders combined with coil embolization was reported in 7 cases. ${ }^{[6]}$

In this case we use a Cerafle ${ }^{\mathrm{TM}}$ atrial septal defect occluder, whose is an alternative to the Amplatzer ${ }^{\text {TM }}$ ASD device with some structural innovations, including a delivery system that allows for free device angulation in relation to the delivery cable. This latter feature may be instrumental for device delivery in this scenario, as the angle between the aorta and pseudoaneurysm was approximately 90 degrees.

An additional challenge in this case was the very large neck of the pseudoaneurysm, as it precludes of treatment with direct thrombin injection or coil embolization as the initial approach and requires large vascular plugs or septal occluder devices. The present case represents one of the largest neck pseudoaneurysm treated percutaneously reported in the literature.

\section{Conclusion}

The use of occluder devices for correction of ascending aorta pseudoaneurysms is an attractive alternative for the treatment of this complex and potential letal complication. Although the existing literature mostly consists of case reports or small series, this strategy may be preferential in patients with a high or prohibitive surgical risk.

\section{ACKNOWLEDGeMents}

The authors thank our patient for permission to publish the case.

\section{CONFLicts OF INTEREST Disclosure}

The authors declare they have no conflicts of interest.

\section{REFERENCES}

[1] Quevedo HC, Santiago-Trinidad R, Castellano J, et al. Systematic Review of Interventions to Repair Ascending Aortic Pseudoaneurysms. Ochsner J. 2014; 14(4): 576-85.

[2] Malvindi PG, Putte BPv, Heijmen RH, et al. Reoperations for aortic false aneurysms after cardiac surgery. Ann Thorac Surg. 2010; 90: 1437-43. PMid:20971235. https://doi.org/10.1016/j.atho racsur. 2010.06 .103

[3] Tang L, Lesser JR, Gössl M, et al. Transcatheter Closure of Complex Ascending Aortic Pseudoaneurysms After Cardiac Surgery. Circ Cardiovasc Interv. 2018; 11: e:007052.
[4] Vallabhajosyula P, Gottret JP, Bavaria JE, et al. Endovascular repair of the ascending aorta in patients at high risk for open repair J Thorac Cardiovasc Surg. 2015; 149: S144-50. PMid:25218530. https://doi.org/10.1016/j.jtcvs.2014.07.063

[5] Bashir F, Quaife R, Carroll JD. Percutaneous closure of ascending aortic pseudoaneurysm using Amplatzer septal occluder device: The first clinical case report and literature review. Catheter Cardiovasc Interv. 2005; 65(4): 547-51.

[6] Lyen SM, Rodrigues JC, Manghat NE, et al. Endovascular closure of thoracic aortic pseudoaneurysms: A combined device occlusion and coil embolization technique in patients unsuitable for surgery or stenting. Catheter Cardiovasc Interv. 2016; 88(7): 1155-69. PMid:27141915. https ://doi.org/10.1002/ccd. 26558 